\title{
Mieloma múltiple. Experiencia de 9 años en el Hospital Naval Almirante Nef
}

\author{
Multiple myeloma. Nine years' experience at the Hospital Naval Almirante Nef \\ Magdalena P. Cortés ${ }^{1,2^{*}}$, Carolina Figueroa ${ }^{3}$, Roxana Varela ${ }^{1}$, Karla Valdivia-Cortés ${ }^{4}$, Katherine Barría ${ }^{5}$, Carla Salvo ${ }^{5}$
}

\begin{abstract}
Resumen
Introducción: el mieloma múltiple (MM) continúa siendo una enfermedad incurable sin embargo, el trasplante autólogo de médula ósea (TAH), y las drogas antineoplásicas han permitido mejorar la sobrevida global (SG) de los pacientes. Materiales y métodos: estudio de cohorte retrospectivo de 50 pacientes con diagnóstico de MM en el hospital Naval Almirante Nef, desde 2005 a 2013. Los pacientes se dividieron en dos cohortes, según la eligibilidad a trasplante, y analizados acordes a la primera línea de tratamiento y la sobrevida global (SG) hasta abril de 2019. Resultados: mediana de edad 73 años (47-88 años), SG 49 meses, y 50\% en etapa-ll del Sistema de Etapificación Internacional. La SG de los 39 no candidatos a TAH fue 46 meses; con un mayor número de respuestas completas y sobrevida, con el esquema melfalán-prednisona-talidomida. La SG de los 11 candidatos a TAH fue 66 meses, siendo el esquema bortezomib-ciclofosfamida-dexametasona el que concentró un mayor número de respuestas completas libres de progresión. Se trasplantó el $45 \%$ de los candidatos, con una mediana de sobrevida de 79 meses versus a los 51 meses de aquellos no trasplantados. Tres casos de neuropatía asociada a talidomida y uno a bortezomib. La SG a los seis meses y a los cinco años de todos los pacientes fue $86 \%$ y $44 \%$, respectivamente. Conclusión: la incorporación de nuevos fármacos permitió obtener mejores resultados de sobrevida lo que se condice con estudios nacionales e internacionales.
\end{abstract}

Palabras clave: mieloma múltiple; talidomida; bortezomib; trasplante autólogo; sobrevida global.

\begin{abstract}
Introduction: Multiple myeloma (MM) is still an incurable disease however, autologous stem cell transplantation (ASCT), and antineoplastic drugs have allowed improving the overall survival (OS) of patients. Materials and methods: A retrospective cohort study of 50 patients diagnosed with MM at the Hospital Naval Almirante Nef, from 2005 to 2013. The patients were divided into two cohorts according to transplantation eligibility and analyzed about first-line treatment and overall survival (OS) up to April 2019. Results: Median age 73 years (47-88 years), OS 49 months, and 50\% in stage-II International Staging System. OS of the 39 non-candidates for ASCT was 46 months: with a higher number of complete responses and survival, with the melphalan-prednisone-thalidomide scheme. The OS of the 11 candidates for ASCT was 66 months, with the bortezomib-cyclophosphamide-dexamethasone scheme being the one with the highest number of progression-free complete responses. Forty-five percent of the candidates were transplanted, with a median survival of 79 months versus 51 months for those not transplanted. Three cases of neuropathy were associated with thalidomide and one with bortezomib. OS at six months and five years for all patients was $86 \%$ and $44 \%$, respectively. Conclusion: The incorporation of new drugs allowed to obtain better survival results, which is by national and international studies.
\end{abstract}

Keywords: multiple myeloma; thalidomide; bortezomib; autologous transplantation; overall survival.

\section{Introducción}

El mieloma múltiple (MM), prototipo maligno de las gammapatías monoclonales, continúa siendo una enfermedad incurable a pesar de los avances terapéuticos. Sin embargo, el trasplante autólogo de células hematopoyéticas (TAH) e incorporación de talidomida,

(1) Escuela de Química y Farmacia, Facultad de Farmacia, Universidad de Valparaíso, Valparaíso, Chile.

(2) Centro de Investigación Farmacopea Chilena, Valparaíso, Chile.

(3) Programa de Magíster en Análisis Clínico, Universidad de Valparaíso, Valparaíso, Chile.

(4) Escuela de Medicina, Facultad de Medicina, Universidad de Valparaíso, Valparaíso, Chile.

(5) Hospital Naval Almirante Nef, Viña del Mar, Chile.

*Autor de correspondencia: magdalena.cortes@uv.cl 
así como bortezomib han permitido mejorar la sobrevida de los pacientes, convirtiéndola así, en una enfermedad más abordable (Rajkumar \& Kumar, 2016; Moreau et al., 2017). El MM es una neoplasia de células plasmáticas en médula ósea que clínicamente se caracteriza por la presencia de hipercalcemia, falla renal, anemia y/o lesiones óseas conocida como CRAB por sus siglas en inglés (Rajkumar \& Kumar, 2016).

El MM, representa entre el $1-2 \%$ de todas las neoplasias, el $17 \%$ de las hematopatías malignas en Estados Unidos, y es la segunda neoplasia hematológica más frecuente (Siegel et al,. 2020). En América Latina, se sabe relativamente poco sobre su incidencia, las características clínicas y los resultados del MM. Recientemente, un estudio multicéntrico de 23 instituciones de Latinoamérica reportó un estudio observacional prospectivo-retrospectivo de MM analizando las respuestas al TAH (Hungria et al., 2017). Los autores del trabajo sugieren que los resultados de los pacientes pueden variar de un país a otro y de una región a otra como consecuencia de las diferentes características biológicas, el estadio en el que se presenta la enfermedad y la disponibilidad de nuevas terapias.

En Chile, se reportó la experiencia de 22 años de TAH en pacientes con MM, entre 1992 y 2014, observando una SG de 55\% de los pacientes trasplantado a los 5 años (Sarmiento et al. 2014). El año 2018, el Programa Adulto Nacional de Drogas Antineoplásicas (PANDA), caracterizó 1103 pacientes con MM tratados con talidomida desde el 2007, el mayor grupo estudiado a la fecha, tratados en el sistema público (Peña et al., 2018). A fines del 2019, el ministerio de salud de Chile incorpora esta patología al plan de garantías explícitas en salud (GES) con el objetivo de garantizar el acceso a su diagnóstico y tratamiento en el sistema público y privado de salud, incluyendo dentro de sus acápites la protección financiera según el nivel de ingresos y tipo de previsión de los usuarios (MINSAL, 2019). Esta estrategia no incluye a las fuerzas armadas, que se rigen por un sistema diferente en relación a financiamiento y proveedores propios (Becerril-Montekio et al., 2011)

En el hospital Naval Almirante Nef (HNAN), se reportó la casuística de MM y gammapatía monoclonal de significado incierto, entre los años 2000 y 2004, y se enfatizó la importancia del registro sistemático de la gammapatía monoclonal de significado incierto como contribución fundamental a la comprensión de su posible evolución a MM (Barría et al., 2010; Cortés et al., 2013). Posteriormente, se incorpora al arsenal terapéutico del MM, talidomida el año 2007, y bortezomib el año 2010. El objetivo del presente trabajo es analizar los resultados terapéuticos en pacientes con MM atendidos en un hospital de las Fuerzas Armadas, y analizar SG según los diferentes esquemas de inducción hasta abril de 2019.

\section{Materiales y métodos}

\section{Caracterización de los pacientes}

Estudio de cohorte retrospectivo de 50 pacientes con diagnóstico de $\mathrm{MM}$, identificados a partir de la revisión retrospectiva de resultados de inmunofijaciones de proteínas de suero y orina, realizadas entre enero del año 2005 y diciembre de 2013, en el HNAN. Se excluyeron los pacientes con diagnóstico distinto a $\mathrm{MM}$, diagnosticados $\mathrm{y} / \mathrm{o}$ tratados en otro establecimiento $\mathrm{y} / \mathrm{o}$ en otro periodo de estudio, o sin consentimiento informado. EI diagnóstico de MM se fundamentó en la presencia de proteína monoclonal (proteína-M) en suero y/u orina, plasmocitosis monoclonal en médula ósea o plasmocitoma, y disfunción orgánica en relación con el mieloma (CRAB), u otros como hiperviscosidad sintomática, amiloidosis e infecciones bacterianas recurrentes, al momento del diagnóstico (International Myeloma Working Group, 2003). A partir de la historia clínica de los pacientes se obtuvieron: datos demográficos, signos y síntomas clínicos, proteína-M, resultados de exámenes de laboratorio al momento diagnóstico, y etapa clínica por el International Stating System (ISS) (Greipp et al., 2005).

\section{Esquemas de tratamiento de inducción}

Candidatos a TAH: pacientes menores de 65 años y sin comorbilidades significativas, fueron tratados con esquemas convencionales de inducción, vincristina, doxorrubicina y dexametasona (VAD) hasta el año 2007, donde se incorporó la combinación talidomida y dexametasona (TD). Desde el año 2010 aquellos pacientes en buen estado funcional se trataron con la asociación bortezomib, ciclofosfamida y dexametasona (CyBorD). A partir el año 2012, ciclofosfamida, talidomida y dexametasona (CTD) se incorporó como esquema de primera línea. Los pacientes que lograron una respuesta completa (RC) o respuesta parcial (RP) muy buena con el tratamiento de inducción realizaron TAH.

No candidatos a TAH: en pacientes mayores de 65 años, se usó como tratamiento de inducción estándar la combinación melfalán y prednisona (MP), con la posterior asociación a talidomida (MPT). Los pacientes que debutaron con una complicación como hipercalcemia, fractura patológica o insuficiencia renal, evidenciando gran masa tumoral, iniciaron con quimioterapia de urgencia con esquemas basados en dexametasona asociada a: vincristina, doxorrubicina, melfalán, bortezomib o talidomida. Otros tratamientos fueron la combinación melfalán y dexametasona (MD) y la combinación bortezomib más dexametasona (VD). Como tratamiento de mantención para pacientes con RP se indicó talidomida de manera continua (50-100 mg/día). 
De acuerdo con el protocolo institucional, basado en recomendaciones de la Sociedad Chilena de Hematología de la época (SOCHIHEM, 2009), se utilizaron los esquemas de inducción presentados en la Tabla 1. Se consignó un registro respecto al tratamiento de inducción, refractariedad, reacciones adversas a medicamentos (RAM), y criterios de respuesta al tratamiento basados en Durie et al. (2006).

Tabla 1: Esquemas de inducción utilizados en pacientes con MM

\begin{tabular}{|c|c|c|}
\hline Esquema & Dosis & Ciclos \\
\hline VAD & $\begin{array}{l}\text { Vincristina: 0,5 mg/día, días 1-4 } \\
\text { Doxorrubicina: } 9 \mathrm{mg} / \mathrm{m}^{2} \text {, días } 1-4 \\
\text { Dexametasona: } 40 \mathrm{mg} / \mathrm{día} \text {, días } 1-4,9-12 \text { y } 17-20\end{array}$ & $\begin{array}{l}\text { 2-4 de } 28 \\
\text { días }\end{array}$ \\
\hline TD & $\begin{array}{l}\text { Talidomida: } 200 \mathrm{mg} / \mathrm{d} \text { áa, días } 1-28 \\
\text { Dexametasona: } 40 \mathrm{mg} \text {, días } 1-4,9-12 \text { y } 17-20\end{array}$ & $\begin{array}{l}\text { 4-6 de } 28 \\
\text { días }\end{array}$ \\
\hline CyBorD & $\begin{array}{l}\text { Bortezomib: } 1,3 \mathrm{mg} / \mathrm{m}^{2} \text {, días } 1,4,8 \text { y } 11 \\
\text { Ciclofosfamida: } 300 \mathrm{mg} / \mathrm{m}^{2} \text {, días } 1,4,8 \text { y } 11 \\
\text { Dexametasona: } 40 \mathrm{mg} \text {, días 1-4, 9-12 y } 17-20\end{array}$ & $\begin{array}{l}\text { 4-6 de } 21 \\
\text { días }\end{array}$ \\
\hline CTD & $\begin{array}{l}\text { Ciclofosfamida: } 500 \text { mg, días } 1-21 \\
\text { Talidomida: } 50-200 \mathrm{mg} \text {, días 1-21 } \\
\text { Dexametasona: } 40 \mathrm{mg} \text {, días 1-4, 9-12 y 17-20 }\end{array}$ & $\begin{array}{l}\text { 4-6 de } 21 \\
\text { días }\end{array}$ \\
\hline MP & $\begin{array}{l}\text { Melfalán: } 0,22 \mathrm{mg} / \mathrm{kg} \text {, días } 1-4 \\
\text { Prednisona: } 100 \mathrm{mg} / \mathrm{m}^{2} \text {, días } 1-4\end{array}$ & $\begin{array}{l}\leq 12 \text { de } 28 \\
\quad \text { días }\end{array}$ \\
\hline MPT & $\begin{array}{l}\text { MP: ídem anterior } \\
\text { Talidomida: } 100 \mathrm{mg} / \text { día, días 1-28 }\end{array}$ & $\begin{array}{l}\leq 12 \text { de } 28 \\
\quad \text { días }\end{array}$ \\
\hline MD & $\begin{array}{l}\text { Melfalán: 0,22 mg/kg, días } 1-4 \\
\text { Dexametasona: } 40 \mathrm{mg} \text {, días } 1-4,9-12 \text { y } 17-20\end{array}$ & $\begin{array}{l}\leq 12 \text { de } 28 \\
\text { días }\end{array}$ \\
\hline VD & $\begin{array}{l}\text { Bortezomib: } 1,3 \mathrm{mg} / \mathrm{m}^{2} \text {, días } 1,4,8 \text { y } 11 \\
\text { Dexametasona: } 40 \mathrm{mg} / \text { día, previo y posterior } \\
\text { a bortezomib }\end{array}$ & $\begin{array}{l}\text { 4-6 de } 21 \\
\text { días }\end{array}$ \\
\hline
\end{tabular}

Análisis estadístico Variables cualitativas: frecuencias absolutas y relativas porcentuales. Variables cuantitativas: mediana y rango. La SG se determinó desde el diagnóstico hasta la fecha de fallecimiento o hasta abril 2019 (total 172 meses). Curvas de sobrevida por el método Kaplan \& Meier (1985), y las diferencias entre grupos mediante la prueba de log-rank. Para el análisis estadístico se utilizó el programa GraphPad Prism 6, San Diego, USA. Se consideró un valor $p<0,05$ como diferencia significativa.

\section{Resultados}

Los 50 pacientes identificados con MM en el periodo del estudio se caracterizaron en función de la elegibilidad a trasplante, evidenciando que los pacientes mayores de 65 años presentaron variables clínicas más severas y estadificaciones más avanzadas respecto a los candidatos a TAH (Tabla 2). La mediana de edad de los pacientes al momento diagnóstico fue de 73 años, presentando un discreto predominio del sexo femenino. Un 32\% de los pacientes fue mayor de 80 años y $2 \%$ menor de 50 años. Cinco pacientes no presentaron comorbilidades, mientras el 74\% presentó hipertensión arterial, $38 \%$ diabetes mellitus y $18 \%$ cardiopatía. En seis casos se encontró antecedentes de alguna otra neoplasia. Al momento de consulta, el dolor óseo estuvo presente en el 76\%, la fatiga en 30\% y la pérdida de peso en $22 \%$. La proteína-M fue de clase IgG en el $54 \%$ de los casos, seguida por IgA en $24 \%$ y cadenas livianas en $22 \%$. Destacan las lesiones osteolíticas en el $55 \%$ de los casos y una anemia generalmente normocítica normocrómica. En cuanto a la estadificación del MM, predominó la etapa-II en $50 \%$ según ISS.

Tabla 2: Características demográficas, clínicas y parámetros de laboratorio de los pacientes diagnosticados con MM y clasificados en función de la elegibilidad a trasplante.

\begin{tabular}{|c|c|c|c|c|}
\hline \multicolumn{2}{|c|}{ Variable } & n/total & Candidato a TAH ( $<65$ años) & Pacientes $>65$ años \\
\hline \multicolumn{2}{|c|}{ Hombres/total } & $23 / 50$ & $7 / 11$ & $16 / 39$ \\
\hline \multicolumn{2}{|c|}{$\begin{array}{l}\text { Edad - mediana años (rango) } \\
\quad \leq 65 \text { años }\end{array}$} & $\begin{array}{c}73(47-88) \\
10 / 50 \\
\end{array}$ & $\begin{array}{c}62(47-66)^{8} \\
10 / 11\end{array}$ & $\begin{array}{c}77(66-88) \\
0 / 39 \\
\end{array}$ \\
\hline \multicolumn{2}{|c|}{ Comorbilidades $(\geq 3)$} & $28 / 50$ & $3 / 11$ & $25 / 39$ \\
\hline \multicolumn{2}{|c|}{ Disminución de peso $(\geq 10 \mathrm{Kg})$} & $7 / 50$ & $2 / 11$ & $5 / 39$ \\
\hline \multicolumn{2}{|c|}{ Dolor óseo } & $38 / 50$ & $8 / 11$ & $30 / 39$ \\
\hline \multicolumn{2}{|c|}{ Proteína M } & $\begin{array}{c}27 / 12 / 0 \\
5 / 6 \\
\end{array}$ & $\begin{array}{c}5 / 3 / 0 \\
2 / 1 \\
\end{array}$ & $\begin{array}{c}22 / 9 / 0 \\
3 / 5\end{array}$ \\
\hline \multicolumn{2}{|c|}{ Plasmocitosis medular (>30\%) } & $25 / 50$ & $4 / 11$ & $21 / 39$ \\
\hline $\mathbf{C}$ & Hipercalcemia* $>2,6 \mathrm{mmol} / \mathrm{L}(10,5 \mathrm{mg} / \mathrm{dL})$ & $11 / 50$ & $5 / 11$ & $6 / 39$ \\
\hline $\mathbf{R}$ & Insuficiencia renal $\mathrm{sCr}>177 \mu \mathrm{mol} / \mathrm{L}(2 \mathrm{mg} / \mathrm{dL})$ & $11 / 50$ & $0 / 11$ & $11 / 39$ \\
\hline A & Anemia $\mathrm{Hb}<105 \mathrm{~g} / \mathrm{L}(10,5 \mathrm{~g} / \mathrm{dL})$ & $26 / 50$ & $1 / 11$ & $25 / 39$ \\
\hline B & Lesiones óseas & $27 / 49$ & $7 / 11$ & $20 / 38$ \\
\hline \multicolumn{2}{|c|}{$\beta 2$ microglobulina $>3,5 \mathrm{mg} / \mathrm{L}$} & $25 / 45$ & $4 / 11$ & $21 / 34$ \\
\hline \multicolumn{2}{|c|}{ Albuminemia $<3,5 \mathrm{~g} / \mathrm{dL}(53 \mu \mathrm{mol} / \mathrm{L})$} & $35 / 48$ & $7 / 11$ & $28 / 37$ \\
\hline \multicolumn{2}{|r|}{ Lactato deshidrogenasa > 4050 nkat/L (243 U/L) } & $6 / 42$ & $1 / 11$ & $5 / 31$ \\
\hline \multicolumn{2}{|c|}{$\begin{array}{l}\text { Etapa según ISS } \\
\qquad \text { | / II / III / no clasificado }\end{array}$} & $7 / 25 / 12 / 6$ & $3 / 7 / 1 / 0$ & $4 / 18 / 11 / 6$ \\
\hline
\end{tabular}

$\mathrm{Hb}$ : Hemoglobina; SCR: creatininemia; * Calcio corregido por albuminemia; ${ }^{\circledR}$ Se incluye un paciente de 66 años sin comorbilidades importantes 


\section{No candidatos a TAH}

Los 39 pacientes mayores a 65 años presentaron una mediana de SG de 46 meses. Se dividieron en dos grupos según esquema de inducción, el primero inició con una terapia basada en MP, y el segundo con esquemas basados en dexametasona (Tabla 3). No se contabilizaron dentro de los criterios de respuesta pacientes no adherentes o con suspensión de tratamiento producto de una RAM grado 3. El año 2007, se incorpora al arsenal farmacéutico talidomida, recibiéndolo un $46 \%$ de los pacientes (23/50). El grupo MP presentó mejor respuesta al tratamiento y SG. El esquema MPT (11/39) concentró un mayor número de RC, un bajo número de casos con progresión de la enfermedad y tres pacientes con enfermedad estable con una SG superior a ocho años al finalizar el periodo de observación.

El grupo con esquemas basados en dexametasona debutó con complicaciones, como insuficiencia renal, citopenias importantes y alta carga tumoral, presentando respuestas menos favorables. Un caso tuvo RC libre de progresión asociada a MD, único paciente vivo hasta el término del seguimiento con una SG de 77 meses (3,2 años).

Para aquellos casos de refractariedad al tratamiento inicial, se utilizaron como esquemas alternativos CyBorD, CTD, lenalidomida y dexametasona $(\mathrm{Rd})$ en dosis bajas (20 mg/día) o MP.

Tabla 3: Respuesta al tratamiento con drogas antineoplásicas en pacientes no candidatos a TAH

\begin{tabular}{|c|c|c|c|c|c|c|}
\hline \multicolumn{2}{|l|}{ Tratamiento inicial } & $\mathbf{n}$ & Edad* (rango) & $\begin{array}{c}\text { ISS } \\
\text { I/II/III/nc }\end{array}$ & $\mathrm{RC} / \mathrm{RP} / \mathrm{P}$ & mSG (rango) \\
\hline \multirow{2}{*}{ Esquema basado en MP } & MP & 10 & $\begin{array}{c}82 \\
(70-83)\end{array}$ & 0/7/0/3 & $1 / 5 / 2$ & $\begin{array}{c}69 \\
(5-153)\end{array}$ \\
\hline & MPT & 11 & $\begin{array}{c}75 \\
(70-84)\end{array}$ & $2 / 7 / 2 / 0$ & $5 / 2 / 3$ & $\begin{array}{c}84 \\
(7-134)\end{array}$ \\
\hline \multirow[t]{2}{*}{ Esquema basado en Dexametasona } & $\begin{array}{l}\text { VAD } \\
\text { MD } \\
\text { VD }\end{array}$ & $\begin{array}{c}10 \\
2 \\
1\end{array}$ & $\begin{array}{c}76 \\
(66-88)\end{array}$ & $2 / 3 / 6 / 2$ & $2 / 2 / 8$ & $\begin{array}{c}14 \\
(1-77)\end{array}$ \\
\hline & TD & 5 & $\begin{array}{c}75 \\
(69-84)\end{array}$ & $0 / 1 / 3 / 1$ & $0 / 2 / 1$ & $\begin{array}{c}14 \\
(2-71)\end{array}$ \\
\hline
\end{tabular}

MP: melfalán, prednisona; MPT: melfalán, prednisona, talidomida; VAD: vincristina, doxorrubicina, dexametasona; MD: melfalán, dexametasona; VD: bortezomib, dexametasona; TD: talidomida; dexametasona; n: número de pacientes; ISS: International Staging System; nc: no clasificado; RC: respuesta completa; RP: respuesta parcial; P: progresión de la enfermedad; $\mathbf{m S G}$ : mediana sobrevida global, meses.

\section{Candidatos a TAH}

Los 11 pacientes candidatos a TAH, presentaron una mediana de SG de 66 meses, con un 91\% en etapa-I y etapa-II, por el ISS al momento diagnóstico. Se consideró dentro del grupo a un paciente de 66 años, dado su buen estado funcional y baja carga de comorbilidades. En cuanto a la respuesta al tratamiento de inducción, se observó 36\% de refractariedad (RF), 27\% de RP y un $36 \%$ con RC y el esquema de inducción CyBorD concentró el mayor número de RC libres de progresión (Tabla 4).

De los seis pacientes en los que no se llevó a caboTAH, dos pacientes se encuentran con enfermedad estable al finalizar el periodo de observación y cuatro fallecieron. Cabe destacar que la menor sobrevida fue de un paciente que falleció a causa de un paro cardiorrespiratorio estando en buenas condiciones luego de un mes de terapia con VAD. La sobrevida para los no trasplantados fue 51 meses. Los pacientes que se trasplantaron usaron esquema base de inducción bortezomib o talidomida. De estos, tres pacientes presentaron RC hasta abril de 2019, fecha en que finalizó el presente estudio, uno falleció 8 meses posteriores a TAH, y el otro paciente conservó RC tras repetir el esquema terapéutico de inducción, falleciendo 3 años después. La mediana de SG para los pacientes trasplantados fue de 79 meses, con un tiempo promedio entre el diagnóstico y el autotrasplante de 14,2 meses (rango 10-18 meses).

Respecto al RAM a los diferentes esquemas de inducción, destacaron en grado 1-2, quince casos de hiperglicemia asociada a corticoides, tres casos de neuropatía asociada a talidomida y una a bortezomib, y tres casos de citopenias por melfalán. Mientras que en grado 3, un caso de trombocitopenia por melfalán, dos casos de reacción dermatológica por talidomida y un caso de shock séptico al esquema VD.

La SG de los 50 pacientes con MM, a 6 meses y a 5 años fue $86 \%$ y $44 \%$, respectivamente; mediana de 49 meses (Figura 1a). La SG de los pacientes a 5 años en función del ISS, para la etapa-I, etapa-II, y etapa-III fue de $57 \%, 48 \%$ y $25 \%$, respectivamente (Figura 1 b). Se observó diferencias estadísticamente significativas entre las estadificaciones I vs III y II vs III $(p<0,05)$. 
Cortés et al.

Tabla 4: Respuesta al tratamiento de inducción en pacientes candidatos a TAH

\begin{tabular}{|c|c|c|c|c|c|c|c|c|c|}
\hline TI & $\mathbf{n}$ & $\begin{array}{l}\text { Edad* } \\
\text { (rango) }\end{array}$ & $\begin{array}{c}\text { ISS } \\
\text { I/II/III/nc }\end{array}$ & $\begin{array}{c}\text { Respuesta al TI } \\
\text { RC/RP/RF }\end{array}$ & $\begin{array}{c}\text { Cambio de } \\
\text { terapia } \\
\text { sílno }\end{array}$ & $\begin{array}{l}\text { TAH } \\
\text { Si/no }\end{array}$ & $\begin{array}{c}\text { Respuesta al } \\
\text { TAH } \\
\text { RC/RP/P }\end{array}$ & $\begin{array}{c}\text { Fallecido } \\
\text { TAH/ no } \\
\text { TAH }\end{array}$ & $\begin{array}{c}\text { SG } \\
\text { rango }\end{array}$ \\
\hline VAD & 1 & 60 & $0 / 1 / 0 / 0$ & $0 / 1 / 0$ & $1 / 0$ & $0 / 1$ & 0 & $0 / 1$ & 1 \\
\hline TD & 5 & $\begin{array}{c}62 \\
(57-63)\end{array}$ & $1 / 3 / 1 / 0$ & $1 / 2 / 2$ & $5 / 0$ & $2 / 3$ & $1 / 0 / 1$ & $1 / 3$ & $21-56$ \\
\hline CyBorD & 3 & $\begin{array}{c}55 \\
(47-63)\end{array}$ & $2 / 1 / 0 / 0$ & $3 / 0 / 0$ & $0 / 3$ & $2 / 1$ & $2 / 0 / 0$ & $1 / 0$ & $27-69$ \\
\hline CTD & 2 & $\begin{array}{c}65 \\
(64-66)\end{array}$ & $0 / 2 / 0 / 0$ & $0 / 0 / 2$ & $2 / 1$ & $1 / 1$ & $1 / 0 / 0$ & $0 / 0$ & $25-63$ \\
\hline
\end{tabular}

TI: tratamiento de inducción; VAD: vincristina, doxorrubicina, dexametasona; TD: talidomida; dexametasona; CyBorD: bortezomib, ciclofosfamida, dexametasona; CTD: ciclofosfamida, talidomida, dexametasona; n: número de pacientes; ISS: International Staging System; nc: no clasificado; TAH: trasplante autólogo de células hematopoyéticas; RC: respuesta completa; RP: respuesta parcial; RF: refractario; P: progresión de la enfermedad; SG: sobrevida global, meses. *mediana.
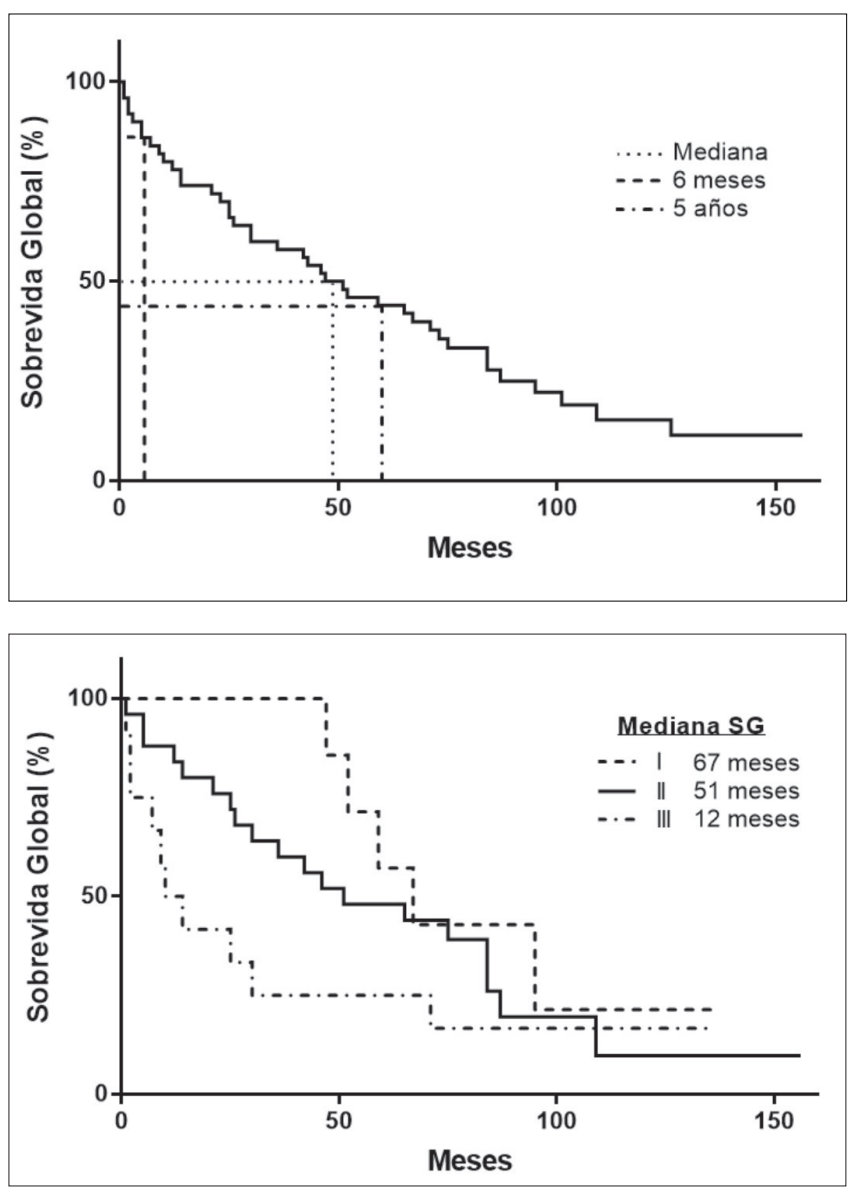

Figura 1: a) Curva de sobrevida global (SG) de los 50 pacientes con MM. b) Curva de SG de los pacientes con MM según International Staging System: etapa-I $(n=7)$, etapa-II $(n=25)$ y etapa-III $(n=12)$. La SG se determinó desde el diagnóstico hasta la fecha de fallecimiento o hasta abril de 2019 (172 meses).

\section{Discusión}

Este es el primer registro de seguimiento farmacológico realizado en pacientes con MM en un Hospital de las Fuerzas Armadas chileno, y con esquemas de tratamientos innovadores, que además de inhibir la proliferación tumoral también actúan bloqueando estímulos del microambiente de la médula ósea que favorecen el desarrollo tumoral. Respecto a los datos demográficos de los pacientes con MM, la mediana de edad diagnóstico fue de 73 años, similar al estudio previo, entre 2000 y 2004 (Barría et al., 2010). Edades más avanzadas, que la reportadas en el sistema público chileno, 66 años ( $n=245)$, entre 1988 y 2002 (Conte et al, 2007); y 64,5 años $(n=1.103)$ por el grupo PANDA, que recopilo datos desde el año 2000 al 2016 (Peña et al, 2018). En este sentido, considerando el estilo de vida activo de la población perteneciente a la Fuerzas Armadas, y que se ha reportado un riesgo dos veces mayor de desarrollar MM en personas sedentarias, se podría explicar porque parte de esta población desarrolla MM en edades más avanzadas (Smith et al., 2015).

A diferencia de lo descrito internacionalmente, se observó un leve predomino de sexo femenino, Kyle et al. (2003) reportaron 59\% hombres de los 1027 pacientes con MM tratados en la Clínica Mayo, entre 1985 y 1998. A nivel nacional, observaron un resultado similar al nuestro, y sugieren que podría deberse a que las pacientes latinoamericanas de sexo femenino, hacen mayor uso del sistema de atención de salud (Peña et al., 2018). Las características clínicas del MM al momento de consulta fueron similares a otros estudios, mientras que la pérdida de peso fue menos común (Kyle et al., 2003; Conte et al., 2007; Hungria et al., 2017). 
La distribución de proteína-M de clase lgG e lgA fue similar a otros estudios (Kyle et al., 2003; Hungria et al., 2017). Mientras que los MM de cadenas livianas libres fueron más frecuentes (22\%), que en estudios nacionales (6\%) (Conte et al., 2007; Peña et al., 2018) y latinoamericanos (13\%) (Hungria et al., 2017). Esto se podría explicar por la mayor disponibilidad de técnicas diagnósticas en el HNAN, tales como la electroforesis de proteínas e inmunofijación de proteínas en suero y orina.

En relación con el ISS, el estudio PANDA mostró que, del total de pacientes con $\mathrm{MM}$, un $68 \%$ están estadificados, atribuyéndolo a que la mayor parte de los centros pertenecientes a la red pública no cuentan con el examen de $\beta 2$-microglobulina; y que la etapa-III es la más frecuente (Peña et al. 2018).

Mientras que, $88 \%$ de los pacientes con MM diagnosticados en el periodo del estudio fueron estadificado según ISS, predominando la etapa II, sugiriendo una pesquisa más temprana debido a los controles periódicos obligatorios de la población naval y el mayor acceso a técnicas diagnósticas.

La SG a los 5 años, para las etapas-I, etapa-II y etapa-III, de $57 \%$, $48 \%$ y $25 \%$, similar a lo proyectado en el sistema internacional de etadificación de MM, 58\%, 42\% y 26\% (Greipp et al., 2005).

En Chile, los pacientes diagnosticados con MM en el sistema público, entre 1988 y 2002, presentaron una mediana de SG de 33 meses $(n=245)$, y fallecen el $20 \%$ a los 6 meses con el arsenal terapéutico de la época (Conte et al. 2007). En el presente estudio, la mediana de SG fue de 49 meses ( $n=50)$, y 14\% fallece a los 6 meses.

\section{No candidatos a TAH}

La talidomida, agente inmunomodulador y antiangiogénico, se incluyó desde 2007 en el estudio PANDA, observando una SG de 30 meses versus 46 meses, en aquellos pacientes que, no versus si recibieron talidomida, respectivamente; e informaron un incrementó en 10\% de la sobrevida a 5 años (Peña et al. 2018). En nuestro estudio, la mediana de SG fue 69 versus 84 meses, en aquellos tratados con esquema MP versus MPT, respectivamente (Tabla 3). De igual manera, estudios internacionales obtiene una mayor cantidad de RC y SG ( Waage et al, 2010; Moreau et al., 2017). Se considera talidomida como parte de la primera línea de tratamiento para pacientes con MM de edades avanzadas, debido a su administración oral y de bajo costo (Latif et al., 2012).

En el otro grupo de pacientes mayores de 65 años que debutaron con evidencia de una gran masa tumoral, el esquema se basó en dexametasona, no observándose una mejor respuesta al tratamiento al incorporar talidomida debido a las características clínicas hetero- géneas y de mayor riesgo del grupo, sumado a la no adherencia al tratamiento. Estas complicaciones son un reto inherente en estos pacientes, asociado a las consecuencias fisiológicas del envejecimiento, las comorbilidades preexistentes y la variada tolerabilidad al tratamiento, siendo un grupo con mayor riesgo de mortalidad temprana y peor supervivencia cuando presentan comorbilidades que limitan el tratamiento (Antoine-Pepeljugoski \& Braunstein, 2019).

\section{Candidatos a TAH}

A la fecha, la elección del tratamiento para un paciente con MM se ha basado en la edad y las comorbilidades. En Chile, el ministerio de salud a través del GES incorpora este tratamiento para menores de 60 años (MINSAL, 2019). En el HNAN, la edad de corte para ser candidato aTAH es 65 años y sin comorbilidades, durante el periodo del estudio, los hematólogos autorizaron excepcionalmente un paciente de 66 años. Sin embargo, la literatura internacional indica la necesidad de evaluar la funcionalidad del paciente por biomarcadores pronóstico y predictivos para determinar la SG, la respuesta al tratamiento y la elección del tratamiento (Pawlyn \& Davies, 2019).

La incorporación de drogas antineoplásicas como el esquema CyBorD, concentraron las mejores respuestas, permitiendo que dos de los tres casos fueran trasplantados, demostrando que la incorporación de inhibidores del proteosoma, como bortezomib, a esquemas basados en inmunomoduladores y corticoides, obtiene mejores resultados ( Rajkumar \& Kumar, 2016; Lei et al., 2019). Todos los pacientes recibieron este esquema por vía endovenosa, que se asocia a una mayor toxicidad neurológica por bortezomib (Lei et al., 2019), observando solo un caso de neuropatía leve. Cabe mencionar, que desde el año 2013 en el HNAN, se comenzó a administrar de manera subcutánea para disminuir dicho efecto.

Del total de candidatos a TAH, $45 \%$ se trasplantó, cifra superior a lo informado en el estudio PANDA, de los pacientes recabados entre los años 2000 y 2016 y que recibieron talidomida a partir de 2007 (Peña et al. 2018). Para los candidatos que sí fueron trasplantados, la mediana de SG fue 79 meses, superior también a lo observado en el mismo estudio, SG 48 meses (Peña et al. 2018).

El grupo que reportó su experiencia de 22 años de trasplante, entre 1992 y 2014, utilizaron diferentes esquemas tratamientos para llevar a RC para realizar el TAH (VAD, MPT, TD y CyBorD), observando una SG de 55\% a los 5 años en los trasplantados (Sarmiento et al., 2014). La sobrevida de nuestros pacientes tratados con TAH a 5 años fue $60 \%$. El tiempo promedio (14 meses) entre el diagnóstico y el trasplante fue mayor a lo reportado (8,5 meses) por el grupo de trasplante, entre 1994 y 2003 (Flores et al., 2005); dado que el HNAN debe derivar a centros especializados para realizar dicha terapia. Respecto a los pacientes no trasplantados, presentaron 
una sobrevida de 51 meses, superior a los 36 meses reportados por Peña et al. (2018); lo que se condice, con la eficacia de los nuevos esquemas terapéuticos, como las estadificaciones de menor riesgo.

Pese a ser parte de las actuales recomendaciones nacionales (SOCHIHEM, 2019) el HNAN no cuenta con determinaciones citogenéticas para categorizar el MM por el Sistema Internacional Revisado (R-ISS). En este punto, es importante recalcar que el perfil genético del tumor es el factor pronóstico más importante, que determina la sobrevida de los pacientes y la respuesta al tratamiento. Adicionalmente, la identificación de nuevos segmentos de la enfermedad basados en la biología del tumor o en el contenido inmunológico del microambiente ofrece una nueva e interesante forma de controlar e incluso erradicar los clones de mieloma, siendo una forma potencial de dirigir el progreso terapéutico hacia la curación en el MM ( Zweegman et al., 2017; Antoine-Pepeljugoski \& Braunstein, 2019; Pawlyn \& Davies 2019). Sin embargo, en la mayoría de los pacientes continuaremos con la inducción, los trasplantes y la terapia de mantención, pero el conocimiento adquirido a nivel internacional ayudará a seleccionar la combinación óptima de fármacos adecuando a la realidad económica y social de cada país.

A pesar del bajo número de pacientes, la ventaja de estudiar un sistema cerrado de salud, con técnicas de diagnósticos idóneas, tratamiento y exámenes de seguimiento asegurado; permite un completo historial de atenciones del paciente. Además, el acceso a tratamientos no disponibles en el sistema público chileno que se ve reflejado en la sobrevida de los pacientes tratados con bortezomib y talidomida. Sin embargo, por ser una revisión que abarca hasta el año 2013, no fueron incluidos pacientes que pueden haber sido tratados con drogas aún más nuevas para TAH, tales como lenalidomida, o daratumumab, que actualmente son parte del arsenal terapéutico en MM (MINSAL, 2020), cuyo financiamiento depende de los recursos de cada institución.

\section{Conclusión}

Los resultados enfatizan la importancia de una población con acceso a los nuevos fármacos asociado a una terapia individualizada con énfasis en la funcionalidad de las personas; siendo trascendente su incorporación al arsenal del servicio público para estrechar las diferencias con los otros sistemas salud.

\section{Reconocimientos}

Al hospital Naval Almirante Nef por la disposición al trabajo colaborativo en pro de mejorar los estándares de la labor asistencial. A los hematólogos, Dr. Armando Peña, Dra. María
Soledad Urquieta, Dra. María de los Ángeles Rodríguez, TM. Gabriela Henríquez y Dra. Isabel Briceño, por su aporte a la investigación. La presente investigación no ha recibido ayudas específicas provenientes de agencias del sector público, sector comercial o entidades sin ánimo de lucro. Finalmente, los autores declaran no tener ningún conflicto de intereses en relación con la investigación presentada.

\section{Contribución de los autores}

Magdalena Cortés Saavedra realizó la concepción de la idea y diseño del estudio. Análisis e interpretación de datos. Redacción y revisión crítica del manuscrito. Aprobación de su versión final.

Carolina Figueroa González efectuó la concepción de la idea y diseño del estudio. Recolección, análisis e interpretación de datos. Redacción del manuscrito. Roxana Varela ejecutó la recolección, análisis e interpretación de datos. Redacción del manuscrito. Karla Valdivia-Cortés desarrolló el análisis e interpretación de datos. Redacción y revisión crítica del manuscrito. Aprobación de su versión final. Katherine Barría Álvarez estableció la concepción de la idea y diseño del estudio. Análisis e interpretación de datos. Revisión crítica del manuscrito. Carla Salvo Porras realizó el aporte de pacientes. Análisis e interpretación de datos. Revisión crítica del manuscrito. Aprobación de su versión final.

\section{Referencias}

Antoine-Pepeljugoski C. \& Braunstein MJ. (2019). Management of Newly Diagnosed Elderly Multiple Myeloma Patients. Current Oncology Reports 21, 64.

Barría K, Maldonado J, Álvarez R, Rodríguez M. \& Cortés MP. (2010). Case Study of Monoclonal Gammopathy of Undetermined Significance and Multiple Myeloma Al'Hospital Naval Almirante Nef', Viña Del Mar, Chile. Revista Medica de Chile 138, 788-790.

Becerril-Montekio V, Reyes JDD. \& Annik M. (2011). Sistema de Salud de Chile. Salud Publica de Mexico 53, S132-143.

Conte G, Figueroa G, Lois V, Cabrera ME, León A, García H. \& Rojas H. (2007). Clinical Features and Survival of Chilean Patients with Multiple Myeloma. Revista Medica de Chile 135, 1111-1117.

Cortés MP, Alvarez R, Maldonado R, Vinet R. \& Barría K. (2013). Monoclonal gammopathy of undetermined significance. En Multiple, pp 111-132 ed. Roman Hajek. Accedido en https://www. intechopen.com/books/multiple-myeloma-a-quick-reflection-onthe-fast-progress el 30 abril 2021. 
Durie BGM, Harousseau JL, Miguel JS, Bladé J, Barlogie B, Anderson K, Gertz M, Dimopoulos M, Westin J, Sonneveld P, Ludwig $H$, Gahrton G, Beksac M, Crowley J, Belch A, Boccadaro M, Cavo M, Turesson I, Joshua D, Vesole D, Kyle R, Tricot G, Alexanian R, Attal M, Merlini G, Powles R, Richardson P, Shimizu K, Tosi P, Rajkumar SV, \& Morgan G. International Myeloma Working Group. (2006). International Uniform Response Criteria for Multiple Myeloma. Leukemia 20, 1467-1473.

Flores C, Conte G, Fardella P, Araos D, Alfaro J, Aravena P, González N \& Larrondo M. (2005). Autotrasplante (AT) de Progenitores Hematopoyéticos En Mieloma Múltiple. Experiencia Clínica. Revista Medica de Chile 133, 887-893.

GraphPad Prism (№ de versión 6). (2015). Windows. San Diego, California USA: GraphPad Software, Inc. Accedido en https://www. graphpad.com/ el 30 abril 2021.

Greipp PR, San Miguel J, Dune BGM, Crowley JJ, Barlogie B, Bladé J, Boccadoro M, Child JA, Harousseau JL, Kyle RA, Lahuerta JJ, Ludwig H, Morgan G, Powles R, Shimizu K, Shustik C, Sonneveld P, Tosi P, Turesson I, \& Westin J. (2005). International Staging System for Multiple Myeloma. Journal of Clinical Oncology 23, 3412-3420.

Hungria VT, Maiolino A, Martinez G, Oliveira Duarte G, Bittencourt R, Peters L, Colleoni G, Oliveira LCO, Crusoé E, Coelho EODM, Pasquini R, Magalhães SMM, Nunes R, Pint. Neto JV, Faria RAO, Souza M, Hamerschlak N, Flantl D, Navarro JR, Conte G, Gomez-Almaguer D, Ruiz-Argüelles G \& Durie BGM. (2017). Observational Study of Multiple Myeloma in Latin America. Annals of Hematology 96, 65-72.

International Myeloma Working Group. (2003). Criteria for the Classification of Monoclonal Gammopathies, Multiple Myeloma and Related Disorders: A Report of the International Myeloma Working Group. British Journal of Haematology 121, 749-57.

Kaplan EL. \& Meier P. (1985). Nonparametric estimation from incomplete observations. JASA, 53, 457-481.

Kyle RA, Gertz MA, Witzig TE, Lust JA, Lacy MQ, Dispenzieri A, Fonseca R, Rajkumar SV, Offord JR, Larson DR, Plevak ME, Therneau TM, \& Greipp PR. (2003). Review of 1027 Patients with Newly Diagnosed Multiple Myeloma. Mayo Clinic Proceedings 78, 21-33.

Latif T, Chauhan N, Han R, Moran A. \& Usmani SZ. (2012). Thalidomide and Its Analogues in the Treatment of Multiple Myeloma. Experimental Hematology \& Oncology 1, 27.
Lei M, Kim EB, Branagan A, Lou U, Zemel M. \& Raje N. (2019). Current Management and Emerging Treatment Strategies for Multiple Myeloma. Rinsho Ketsueki 60, 1243-1256.

MINSAL (Ministerio de Salud de Chile). (2019). Decreto Supremo №22. Aprueba Garantías Explícitas En Salud Del Regímen General de Garantías En Salud. Accedido en https://www.bcn.cl/leychile/ el 30 abril 2021.

MINSAL (Ministerio de Salud de Chile). (2020). Protocolo clínico Mieloma Múltiple y otras gammapatías monoclonales. Accedido en https://www.sochihem.cl el 30 mayo 2021.

Moreau P, San Miguel J, Sonneveld P, Mateos MV, Zamagni E, Avet-Loiseau H, Hajek R, Dimopoulos MA, Ludwig H, Einsele H, Zweegman S, Facon T, CavoM , Terpos E, Goldschmidt H, Attal M. \& Buske C. (2017). Multiple Myeloma: ESMO Clinical Practice Guidelines for Diagnosis, Treatment and Follow-Up. Annals of Oncology 28, 52-61.

Pawlyn C. \& Davies FE. (2019). Toward Personalized Treatment in Multiple Myeloma Based on Molecular Characteristics. Blood 133, 660-675.

Peña C, Rojas C, Rojas H, Soto P, Cardemil D, Aranda S, Contreras C, La Roca G, Russo M, Pérez C. \& Lois V. (2018). Survival of 1,103 Chilean Patients with Multiple Myeloma Receiving Different Therapeutic Protocols from 2000 to 2016. Revista Médica de Chile 146, 869-875.

Rajkumar SV. \& Kumar S. (2016). Multiple Myeloma Diagnosis and Treatment. Mayo Clinic Proceedings 91,101-19.

Sarmiento M, Lira P, Ocqueteau M, Rodríguez MA, García MJ, Jara V, Bertín P, \& Ramírez P. (2014). Autologous Hematopoietic Cell Transplantation in Patients with Multiple Myeloma. Experience in 53 Patients. Revista Medica de Chile 142,1497-1501.

Siegel RL, Miller KD. \& Jemal A. (2020). Cancer Statistics, 2020. CA: A Cancer Journal for Clinicians 70, 7-30.

Smith L, McCourt O, Henrich M, Paton B, Yong K, Wardle J. \& Fisher A. (2015). Multiple Myeloma and Physical Activity: A Scoping Review. BMJ Open 5, e009576.

SOCHIHEM (Sociedad Chilena de Hematología). (2009). Recomendaciones clínicas mínimas para el diagnóstica y tratamiento de Mieloma Múltiple. Accedido en https://www.sochihem.cl/ el 30 abril 2021.

SOCHIHEM (Sociedad Chilena de Hematología). (2019). Guías prácticas clínicas para diagnóstico y tratamiento del Mieloma Múltiple. 1-50. Accedido en https://www.hematologia.org el 09 diciembre 2019. 


\section{Cortés et al.}

Waage A, Palumbo A, Fayers P, Beksac M, Hulin C, Bringhen J, Sonneveld P, Wijermans P, Facon T, NMSG, GIMEMA, TMSG, HOVON, IFM. \& European Myeloma Network. (2010). MP versus MPT for Previously Untreated Elderly Patients with Multiple Myeloma: A Meta-Analysis of 1,682 Individual Patient Data from Six Randomized Clinical Trials. J Clin Oncol 28, 8130-30.
Zweegman S, Engelhardt M. \& Larocca A. (2017). Elderly Patients with Multiple Myeloma:Towards a Frailty Approach? Current Opinion in Oncology 29, 315-21. 